\title{
REPENSER LA CITOYENNETÉ. RACE ET GENRE DANS LES RÉVOLUTIONS HISPANO-AMÉRICAINES
}

\section{Federica Morelli}

Belin | « Clio. Femmes, Genre, Histoire »

2021/1 n 53 | pages 127 à 149

ISSN 1252-7017

DOI 10.4000/clio.19770

Article disponible en ligne à l'adresse :

https://www.cairn.info/revue-clio-femmes-genre-histoire-2021-1-page-127.htm

Distribution électronique Cairn.info pour Belin.

(C) Belin. Tous droits réservés pour tous pays.

La reproduction ou représentation de cet article, notamment par photocopie, n'est autorisée que dans les limites des conditions générales d'utilisation du site ou, le cas échéant, des conditions générales de la licence souscrite par votre établissement. Toute autre reproduction ou représentation, en tout ou partie, sous quelque forme et de quelque manière que ce soit, est interdite sauf accord préalable et écrit de l'éditeur, en dehors des cas prévus par la législation en vigueur en France. Il est précisé que son stockage dans une base de données est également interdit. 


\section{Clio. Femmes, Genre, Histoire}

$53 \mid 2021$

Le genre des indépendances

\section{Repenser la citoyenneté. Race et genre dans les révolutions hispano-américaines}

Citizenship, race and gender in the Hispano-American revolutions

Repensar la ciudadanía. Raza y género en las revoluciones hispanoamericanas

\section{Federica Morelli}

\section{(2) OpenEdition}

\section{Journals}

Édition électronique

URL : https://journals.openedition.org/clio/19770

DOI : 10.4000/clio.19770

ISSN : $1777-5299$

\section{Éditeur}

Belin

Édition imprimée

Date de publication : 1 juillet 2021

Pagination : 127-149

ISSN : 1252-7017

Distribution électronique Cairn

\section{CAIRN INFO}

CHERCHER, REPÉRER, AVANCER

\section{Référence électronique}

Federica Morelli, «Repenser la citoyenneté. Race et genre dans les révolutions hispano-américaines », Clio. Femmes, Genre, Histoire [En ligne], 53 | 2021, mis en ligne le 02 janvier 2024, consulté le 04 août 2021. URL : http://journals.openedition.org/clio/19770 ; DOI : https://doi.org/10.4000/clio.19770 


\section{Actualité de la recherche}

\section{Repenser la citoyenneté. Race et genre dans les révolutions hispano-américaines}

Federica MORELLI

Cet article prend en compte la question du genre à l'époque des Indépendances dans l'Amérique hispanique à partir des recherches les plus récentes sur les descendants d'Africains et les femmes, en raison de la forte relation entre race et genre, souvent relevée à cette époque par les contemporains eux-mêmes. À l'encontre de l'hypothèse qui considère le statut juridique et social de «la» femme comme immuable, on soulignera les ruptures entre la fin de l'époque coloniale et la période des Indépendances quant aux droits des femmes et à leur inclusion dans la citoyenneté.

On connait de mieux en mieux désormais les efforts des AfroAméricains pour accéder aux droits de citoyenneté à l'époque des révolutions atlantiques : ils ne se limitèrent pas aux seules actions les plus visibles dans la sphère publique. Les femmes, comme les esclaves, étaient en principe exclus des droits de citoyenneté en raison de leur dépendance vis-à-vis du maittre ou du paterfamilias. Cependant, ils utilisèrent les nouveaux principes constitutionnels et les espaces de manœuvre laissés par les systèmes juridiques pour entamer des actions et revendiquer leurs droits.

Après avoir mis en évidence comment évolue l'historiographie à propos de la participation des femmes à l'Indépendance au cours de 
ces dernières décennies, on montrera que, suite aux travaux sur la participation des esclaves et des libres de couleur aux mouvements politiques et militaires, une nouvelle approche par le genre peut être poursuivie. Ensuite, on analysera les moyens par lesquels les femmes pouvaient réclamer leurs droits, et donc transformer leur position dans la société, à la fin de l'époque coloniale ou pendant la période des Indépendances.

\section{L'historiographie autour des femmes et des Indépendances}

Les études sur la participation des femmes aux guerres d'Indépendance ont une longue tradition en Amérique latine, qui remonte au début du $\mathrm{XX}^{\mathrm{e}}$ siècle. Adoptant une approche typiquement patriotique, ces travaux célèbrent les femmes qui ont assisté les hommes patriotes ou qui ont été victimes des royalistes. La majorité de ces dernières venaient des couches les plus élevées de la société, se distinguant comme mères, épouses ou amantes d'hommes bien plus célèbres. Dans cette narration, les femmes qui soutenaient les royalistes ont été délibérément négligées; de la même manière, les femmes aimaient et se sacrifiaient, mais elles n'apparaissent jamais comme protagonistes, porteuses de leurs opinions politiques ${ }^{1}$.

Cette approche a changé autour des années 1980 avec la fin des dictatures puis l'essor des mouvements féministes en Amérique latine. Des travaux importants, comme ceux de Asunción Lavrin, Silvia Arrom et d'autres historiennes, ont alors examiné la question du genre dans un contexte théorique et chronologique plus ample ${ }^{2}$. Sur la base de ces premiers travaux, des études ont récemment abordé les thèmes du genre, de la sexualité, de la famille et de la vie quotidienne des femmes, en s'appuyant sur des documents judiciaires concernant les divorces, l'adultère, le concubinage, la bigamie, l'inceste, les conflits parents-enfants pour les choix autour du mariage, la dispense de consanguinité. Se concentrant sur les pratiques quotidiennes et leurs valeurs morales, ces études ont révélé un certain écart entre les pratiques et les prescriptions légales et religieuses des sociétés

Voir, par exemple, Carranza 1910 ; Cartula Brú 1945.

2 Lavrin 1978, 1984, 1989 ; Arrom 1985 ; Franco 1989 ; Masiello 1992 ; Fletcher 1994. 
coloniales. Pourtant, elles refusent l'idée selon laquelle l'idéologie du genre est toujours imposée par le haut et rejetée ou négociée par le bas; elles ont plutôt démontré que le genre est continuellement construit par l'interaction entre ceux qui possèdent et ne possèdent pas le pouvoir ${ }^{3}$.

Ces recherches ont transformé l'historiographie sur le rôle des femmes dans les Indépendances hispano-américaines mais elles n'ont pas réussi pour autant à proposer un nouveau modèle pour repenser le genre à cette époque de rupture politique et sociale. Même si par rapport au passé certaines idées ont radicalement changé, le rôle des femmes est encore pensé comme enfermé dans une relation de type patriarcal. En dépit de leur participation aux guerres, la plupart des historiens continuent à négliger l'apport de ces femmes dans la mise en cause des structures patriarcales.

Malgré cette faiblesse de fond, le résultat le plus novateur à propos du rôle des femmes dans les mouvements pour l'Indépendance a été, en 2001, la construction d'une importante base de données, Gender and Latin American Independence ${ }^{4}$. Elle montre l'ample participation des femmes dans les luttes pour les Indépendances dans l'Amérique espagnole et portugaise de la première moitié du XIX $^{\mathrm{e}}$ siècle $^{5}$. Accessible en anglais, espagnol et portugais, cet instrument formidable contient les noms et les biographies des femmes, tirées des sources documentaires des archives nationales de différents pays latino-américains ainsi que des sources secondaires. Genderlatam inclut les données de 2300 personnes, 3900 événements (naissances, mariages, morts, luttes, récompenses, emprisonnements) et 340 images.

3 Johnson \& Lipsett-Rivera 1998; Chambers 1999; Zegarra 1999; Dore \& Molyneux 2000 ; Ramírez 2000 ; Rodríguez Sáenz 2000 ; Socolow 2000 ; UribeUrán 2000.

4 https://www.nottingham.ac.uk/genderlatam/.

5 Le projet est hébergé à l'université de Nottingham et a été financé par la même université et le UK Arts and Humanities Research Council (AHRC) entre 2001 et 2014. À l'occasion du bicentenaire des indépendances hispano-américaines, il a été conçu pour repenser les Indépendances en termes de genre. Même si le projet repose sur la collaboration de chercheuses de l'Amérique latine, il a été piloté par des chercheurs anglais. 
Cette base de données bouleverse l'image des femmes dans les Indépendances par rapport aux recherches antérieures. Dans un premier temps, on découvre que les femmes ont participé aux mouvements indépendantistes aussi bien du côté des royalistes que des patriotes. On apprend par exemple que les femmes royalistes de Mexico avaient créé une organisation de 2500 membres pour vénérer la vierge de los Remedios, la sainte patronne de l'armée royaliste, en défiant les rebelles dont la sainte patronne était la vierge de Guadalupe. En second lieu, la présence des femmes sur les champs de bataille ne se limitait pas à soigner ou cuisiner pour les soldats. Elles prenaient aussi part aux batailles, comme par exemple Juana de Azurduy, métisse et lieutenant-colonel, épouse de Manuel Padilla, qui a conduit avec son époux une armée de 6000 soldats dans le Haut Pérou. Elle dirigeait son propre bataillon de femmes, appelées les « loyales », qui participa à seize conflits ${ }^{6}$.

Malgré les transformations de l'historiographie latinoaméricaniste sur le genre, dont cette base de données, il reste des lacunes importantes à combler pour ce qui est de la construction du genre. En dépit du rôle important joué par les femmes dans les mouvements d'Indépendance, la plupart des études tendent à souligner une forte continuité entre l'époque coloniale et la nouvelle ère républicaine, quant à la soumission des femmes aux hommes, leur confinement dans la sphère domestique, l'idéologie patriarcale. L'absence d'un mouvement public et visible de revendications des droits et une lecture un peu trop sommaire des sources juridiques ont amené l'historiographie latino-américaniste à affirmer que même si les femmes ont participé aux guerres, elles furent largement exclues de la sphère politique et de la citoyenneté durant et après l'indépendance, en continuité avec la période coloniale? ${ }^{7}$ Les études sur le langage politique avec une perspective de genre confirment cet aspect, en démontrant que la différence sexuelle offre un contretype à l'idéal masculin. Alors que le caractère efféminé était associé à la dégénérescence, à la non-violence, à la paix, à la

6 https://www.nottingham.ac.uk/genderlatam/database/wp/

7 Il ne s'agit seulement pas d'historiens latino-américains mais aussi d'historiens étrangers travaillant sur l'Amérique latine. Voir, par exemple, Zahler 2015. 
dépendance et à la soumission, la masculinité hégémonique, qui avait besoin d'être constamment réaffirmée, était par contre attribuée à la capacité des hommes de protéger les autres dépendants, surtout les femmes et les enfants ${ }^{8}$.

Or, cette manière de penser la relation entre genre et politique se fonde sur une série de sources qui concernent d'un côté les débats politiques, lois et constitutions et, de l'autre, les biographies, écrits ou correspondances des femmes appartenant aux élites. Si on change d'approche méthodologique en analysant les sources judiciaires et le recours des femmes devant les tribunaux, la perspective évolue sensiblement. En ce sens, les historiens du genre devraient suivre le parcours ouvert par les historiens de la race qui ont récemment renouvelé le rôle joué par les descendants des Africains - libres et esclaves - dans la colonie et l'Indépendance en utilisant les archives judiciaires.

\section{Droits, race et genre}

Aux cours de ces dernières années les études sur les guerres d'Indépendance de l'Amérique espagnole ont été radicalement renouvelées. Au lieu de se concentrer sur les libertadores, sur les élites politiques et militaires ou les transformations essentiellement politiques (la création des juntes, la convocation des Cortès de Cadix, les déclarations d'Indépendance, les nouvelles constitutions), elles ont consacré plus d'attention à la relation entre guerre et société, à la manière dont les sociétés locales étaient affectées par la violence, au recrutement forcé des soldats et aux déplacements des populations, à la manière dont la participation des groupes subalternes aux armées a changé leur statut politique et social ${ }^{9}$. Dans ce contexte, les personnes d'ascendance africaine (esclaves et libres) ont acquis un rôle extrêmement important : non seulement ils se sont révélés décisifs pour la défaite des Espagnols, mais leur participation aux guerres a changé leur position sociale et politique ${ }^{10}$.

\footnotetext{
8 Dudink \& Hagemann 2004 ; Chambers 2006.

9 Thibaud 2003; Rabinovich 2013 ; Ortiz Escamilla 2014.

10 Helg 2004 ; Blanchard 2008 ; Lasso 2007.
} 
La découverte de cette dimension négligée pendant longtemps par l'historiographie, a introduit la question raciale dans le cadre des Indépendances de l'Amérique espagnole. Ces recherches ont démontré que les personnes d'ascendance africaine luttaient pour la reconnaissance de leurs droits autant sur les champs de bataille que devant les tribunaux. Le langage de la liberté et de l'égalité, diffusé par les révolutions atlantiques, était revendiqué par les esclaves et castas dès la fin de l'époque coloniale ${ }^{11}$.

En même temps, les historiens de l'espace atlantique ont remarqué que les esclaves entretenaient un lien fort et continu avec le système juridique des différents espaces coloniaux. Au cours des dernières décennies, on a étudié comment, à travers les recours aux tribunaux ou la participation à des procès civils ou criminels, les esclaves ont participé activement à la création des concepts juridiques, coutumes, institutions et droit ${ }^{12}$. Contrairement au passé, lorsque l'étude des systèmes esclavagistes concernait les codes juridiques, les statuts et les doctrines, les historiens d'aujourd'hui ne considèrent plus la loi comme un ensemble fixe de principes et préceptes, mais plutôt comme un espace politique et social négocié dans lequel différents intérêts, parmi lesquels ceux des esclaves, entrent en conflit.

Fondée sur les sources juridiques et une échelle micro cette approche peut contribuer à aborder la question du genre pendant l'Indépendance sous un nouveau jour. La tendance à voir les femmes comme subordonnées et enfermées dans la sphère privée a été en effet radicalement critiquée par l'historiographie du genre dans d'autres contextes. Explorant les frontières de l'appartenance, les formes d'inclusion et d'exclusion inhérentes à la citoyenneté, ces travaux ont distingué le statut légal de la citoyenneté et sa pratique réelle, en soulignant qu'elle concernait non seulement ceux qui étaient citoyens au sens strict, mais aussi ceux qui étaient exclus et s'emparaient du langage de la citoyenneté pour réclamer leurs droits ${ }^{13}$.

\footnotetext{
11 Gómez 2008 ; Soriano 2018.

12 De La Fuente \& Gross 2010.

13 Landes 1992 ; Hufton 1992 ; Cott 1998 ; Kerber 1998.
} 
Certes l'historiographie a longtemps soutenu que les groupes subalternes avaient été exclus de la participation politique dans l'Amérique latine du XIX siècle - les régimes indépendants s'emparant des mécanismes de hiérarchisation et d'exclusion de l'époque coloniale. Toutefois les nouvelles études sur la période ont complètement bouleversé cette image d'une participation restreinte et limitée aux élites créoles. Se libérant de l'idée selon laquelle les expériences libérales latino-américaines représentaient des tentatives avortées d'appliquer les modèles européens à des réalités trop différentes, les derniers travaux ont souligné l'originalité et la radicalité de ces expériences par rapport à d'autres contextes ${ }^{14}$. À l'inverse de l'image que l'on avait des systèmes politiques «oligarchiques», restreints et présentant une faible participation politique, ces travaux ont démontré non seulement que la majorité des États indépendants adoptèrent des conditions de suffrage très larges pour l'époque, mais aussi que la participation politique assumait des formes variables. Surtout, l'opposition entre État et société, qui pendant longtemps a influencé les manières de penser la citoyenneté comme une extension progressive des droits par le haut, n'est plus si nette. Les sociétés locales s'emparent des institutions, les adaptant à leurs nécessités, rompant par-là l'apparente unité du processus normatif revendiqué par l'État. En ce sens, l'analyse des pratiques et des normes autour de la citoyenneté dans l'Amérique latine du XIXe siècle dépasse les stéréotypes qui ont longtemps limité la compréhension des expériences postcoloniales.

Par cette approche les historiens ont découvert que les groupes subalternes, et spécialement ceux d'ascendance africaine, participaient activement à la politique et donc à la citoyenneté. Les sources montrent que malgré leur exclusion du statut des citoyens, établie par la constitution de Cadix en 1812, les libres de couleur participent au suffrage pendant la période libérale espagnole ${ }^{15}$. Il ne s'agissait pas d'une violation des normes constitutionnelles, mais plutôt d'espaces de manœuvre laissés par les normes elles-mêmes aux communautés locales. Le texte constitutionnel en effet n'excluait pas tous ceux qui

\footnotetext{
14 Sábato 2018.

15 Morelli 2020 : chap. III.
} 
possédaient une ascendance africaine du vote, mais seulement ceux qui « étaient réputés et considérés originaires de l'Afrique ${ }^{16}$. La réputation des individus continuait donc à avoir plus d'importance que la couleur de la peau afin de déterminer l'inclusion ou l'exclusion d'un individu du corps politique.

En effet, tant la constitution de 1812 que celles des nouveaux États indépendants, s'inspirèrent de celle de Cadix, dans laquelle le concept de citoyen était très lié à celui de vecindad. La notion de vecino, d'origine ibérique, renvoyait en effet à la réputation sociale de l'individu dans la communauté d'appartenance : s'il était reconnu par les autres comme membre de cette dernière, il était de fait accepté comme citoyen sans besoin d'une légitimation par les autorités étatiques ${ }^{17}$.

En adoptant une définition de la citoyenneté non soumise à des conditions fiscales ou de propriété, l'accès des libres de couleur et des ex-esclaves, aussi bien que des Indiens, à la citoyenneté fut assez large pour l'époque. Leur mobilisation pendant les guerres d'Indépendance s'était avérée déterminante pour la victoire finale sur les Espagnols ; par conséquent, les nouvelles républiques indépendantes ne pouvaient pas les exclure du corps politique qu'ils avaient largement contribué à former. En effet, les études sur la première moitié du $\mathrm{XIX}^{\mathrm{e}}$ siècle révèlent une ample participation des secteurs populaires à la politique, non seulement par le suffrage, mais aussi par les associations et la mobilisation dans les conflits civils. Même si les discriminations contre les Indiens et les descendants des Africains n'ont pas été définitivement supprimées, les guerres ont quand même contraint les républiques hispano-américaines à éliminer les distinctions raciales au moins sur le plan légal. En même temps, un discours puissant contre l'oppression raciale s'était imposé au cours

16 Article 22 de la Constitution de Cadix : «Les Espagnols qui, du côté de leur père ou de leur mère, sont considérés et réputés originaires d'Afrique, pourront mériter et obtenir le titre de citoyen qui sera accordé par les Cortès à ceux d'entre eux qui auront rendu des services éminents à la patrie, ou qui se seront distingués par leurs talents, leur application et leur bonne conduite, pourvu qu'ils soient fils légitimes de pères libres, qu'ils soient mariés avec une femme libre, et domiciliés dans les domaines espagnols, et qu'ils y exercent une profession, un emploi ou quelque genre d'industrie utile, avec un capital à eux appartenant ».

17 Herzog 2003 : 52-61. 
du XIX $\mathrm{X}^{\mathrm{e}}$ siècle, obligeant les dirigeants politiques à négocier avec les subalternes en utilisant le langage de l'égalité et de l'inclusion ${ }^{18}$.

Or, en s'appuyant sur cette littérature et en utilisant des sources différentes, la question du genre et de l'accès des femmes à la citoyenneté pendant l'Indépendance peut être profondément reconsidérée. Ces dernières, en effet, comme les Noirs et les Indiens, étaient exclues de la citoyenneté à cause de leur condition de dépendance. Alors que les premiers étaient exclus sur la base de leur statut servile, les femmes l'étaient en tant que dépendantes du pater familias. La distinction entre droits civils et politiques avait été introduite par le constitutionalisme français dans le but d'abolir la société d'ordres et de corps et faire triompher la figure du citoyenindividu. Cette distinction s'appuyait sur la notion d'« autonomie de la volonté », qui permettait d'identifier ceux qui avaient droit de vote. La participation à la vie politique était en principe réservée aux individus libres et autonomes, les seuls à produire des effets juridiquement recevables ${ }^{19}$.

Selon les libéraux de l'époque, l'absence de l'autonomie de la volonté caractérisait sans aucun doute les esclaves et les femmes. Bien que le texte de la constitution de Cadix n'affirme pas une exclusion évidente des femmes des droits de citoyenneté, le parallèle avec les esclaves est bien présent dans la mentalité des constituants, comme le démontre la réponse du député libéral espagnol, Diego Muñoz Torrero, à la proposition des délégués américains de supprimer l'esclavage :

$[\ldots]$ si on amène très loin ces principes de ce qui est dit être de justice rigoureuse, il serait inévitable d'accorder aux femmes les droits politiques outre les droits civils, les admettre dans les assemblées électorales et dans les mêmes Cortes $^{20}$.

Malgré cela, identifier les personnes ne disposant pas de l'autonomie de la volonté dans des sociétés où les liens corporatifs et personnels étaient multiples et très imbriqués restait délicat. Par exemple, les domestiques étaient exclus du suffrage au titre de leur dépendance vis-à-vis d'un tiers et de leur assimilation à la sphère du

\footnotetext{
18 Lasso 2007 : 57-61.

19 Rosanvallon $1992: 111-112$.

20 Diario de Sesiones de las Cortes Generales y Extraordinarias, 6 septembre 1811, t. 339.
} 
domus, à savoir à la maison et à la famille patriarcale, et non du fait de leurs capacités et de leur cens. La volonté des constituants n'était pas de priver du droit de vote tous les salariés ou travailleurs dépendants d'un propriétaire «dont les services n'étaient pas purement personnels ", mais, comme on l'affirma à Cadix, seulement «les domestiques exclusivement dévoués au service de la personne ${ }^{21}$. Si cela excluait automatiquement les esclaves et les Indiens domestiques des droits politiques, l'exclusion d'autres catégories fut plus ambiguë et objet de débat. Comment fallait-il agir envers les Indiens qui travaillaient dans des haciendas mais qui restaient liés également à leurs communautés, les libres de couleur qui étaient artisans ou commerçants, les femmes veuves ou divorcées qui étaient chefs de famille sans dépendre de quelqu'un?

Les réponses des autorités locales n'étaient pas toujours claires et tranchantes au sujet de l'exclusion de ces catégories. Le processus de revendication de leurs droits à la fin de la période coloniale ainsi que le rôle joué dans les guerres d'Indépendance contribuèrent à créer des incertitudes quant à leur exclusion ou inclusion dans la citoyenneté.

\section{Les droits des femmes à la fin de la colonie}

Les femmes avaient toujours bénéficié de la possibilité de dénoncer leurs maris ou partenaires à la justice dans l'Amérique espagnole. Ces droits étaient établis par des codes, compilations et fueros (privilèges) locaux aussi bien que par le droit canonique et les décisions conciliaires de l'Église hispano-américaine sur le divorcium. Les femmes, donc, avaient toujours eu la possibilité de recourir aux tribunaux ecclésiastiques pour obtenir l'annulation du mariage ou la séparation ${ }^{22}$.

Cependant, comme le souligne Bianca Premo, on assiste dans la seconde moitié du XVIII' siècle à une forte augmentation des recours des femmes devant les tribunaux pour les séparations et les demandes de pension. Les plaintes ne proviennent plus seulement des femmes appartenant aux élites, mais également de celles des couches

\footnotetext{
21 Intervention du député Golfin, Diario de las Sesiones de las Cortes Generales y Extraordinarias, 10 septembre 1811, t. 343.

22 Arrom 1985 ; Seed 1988 ; Klueger 2003 ; Uribe-Uran 2015 : 29-38.
} 
moyennes et basses ${ }^{23}$. Ces transformations ne sont pas seulement le résultat d'une meilleure prise de conscience de leurs droits, mais aussi des politiques des Bourbons, qui en 1787 publient un décret sur la question des pensions, reconnaissant la juridiction royale sur de tels $\operatorname{cas}^{24}$. Cela contribua à changer la jurisprudence sur le mariage qui fut de plus en plus considéré comme un contrat, avec un accent particulier sur le caractère non seulement sacré mais aussi civil des droits et devoirs qu'il impliquait.

Alors qu'auparavant elles demandaient la séparation ou l'annulation du mariage sans remettre en question la position de suprématie des hommes dans la famille et la société, au XVIII e siècle elles commencèrent à contester le pouvoir masculin tant au foyer que dans l'espace judiciaire, réclamant une relation plus égalitaire, dans laquelle hommes et femmes respecteraient leurs obligations réciproques envers la famille. Pour ce faire, devant les tribunaux, les femmes représentaient les mariages comme une forme d'esclavage et se décrivaient comme des esclaves. En tant que dépendantes du pater familias, qui gardait le contrôle sur les personnes et la propriété, femmes et esclaves étaient les victimes du langage et des actions d'une telle dépendance.

C'est particulièrement évident dans les cas de séparation, où les femmes se plaignent que leur mari est violent, infidèle, négligent vis-àvis des besoins matériels de la famille. Ignacia Azcarate, une femme pauvre de Caracas, déclare devant le tribunal ecclésiastique en 1795 :

[...] une femme est donnée à un homme en mariage pour devenir sa partenaire, non pour souffrir vexation, oppression et violence par des hommes irascibles qui, abusant de leur pouvoir et force physique, oublient leurs devoirs principaux comme époux, chrétiens et citoyens ${ }^{25}$.

De la même manière, Juana Feliciana Díaz, avait écrit au tribunal :

Que la condition des femmes mariées est dure! On ne trouve guère un époux qui ait un égal respect pour sa conjointe [...] Le mari devient un

\footnotetext{
23 Premo $2017: 121-123$.

24 Premo $2017: 148-150$.

25 Diaz $2004: 96$.
} 
tyran, l'épouse une esclave, la maison l'enfer et les enfants sont les témoins malheureux de cette horrible et lamentable métamorphose ${ }^{26}$.

En même temps, dans la vice-royauté du Pérou, l'avocat de Margarita Zegarra qualifie son mari de «barbare et inhumain » devant le maire provincial de Lima. Une autre femme de Trujillo affirma que son époux, au lieu d'être le " compagnon d'une seule chair», était «le plus barbare et atroce tyran, me traitant avec des sévices continus comme si j'étais son esclave ${ }^{27}$.

Plus que des accusations pro-forma, les termes de "tyrannie», « despotisme », " déshumanité », « esclavage » représentaient une attaque contre l'autorité patriarcale. En utilisant ce lexique, les pétitions de ces femmes commencèrent donc à faire référence au langage du droit naturel - très diffusé à l'époque dans l'Amérique espagnole - qui liait la tyrannie à la théorie du droit naturel. Ce dernier prévoyait le droit à l'auto-préservation, comme l'indique clairement le recours d'Ana Cárdenas, de la ville de Trujillo, qui, après avoir dénoncé son mariage caractérisé par des "sévices tyranniques » et une "cruauté barbare», affirme que «par droit naturel » elle avait le droit de se séparer de son époux », dès lors que le mariage ne peut pas «se résumer à une vie de captivité tyrannique $»^{28}$.

La reconnaissance pour les femmes du droit à une pension allait dans la même direction, comme le reconnut l'avocat de don José de Eslava (de Tepoztlán au Mexique), poursuivi en justice par sa femme pour obtenir une pension après la séparation. Contestant la demande de la femme, qui avait également exigé le remboursement des frais judiciaires, l'avocat répondit :

[...] les pensions alimentaires sont spécifiquement enracinées dans la justice parce qu'elles sont fondées sur le droit naturel, qui demande la conservation des êtres vivants; la même raison ne s'applique pas aux frais judiciaires car ils sont normalement volontaires (de gracia) ${ }^{29}$.

Le droit aux aliments devint si inhérent au droit naturel qu'en 1800 il fut reconnu par un tribunal civil de Lima à une femme qui avait été

\footnotetext{
26 Díaz 2004 : 96.

27 Premo $2017: 146$.

28 Ibid. : 147.

29 Ibid. : 149.
} 
condamnée pour avoir conspiré avec son amant à l'empoisonnement de son mari. Son droit aux aliments fut confirmé, même en appel ${ }^{30}$.

L'action des femmes dans les tribunaux aussi bien que les changements judiciaires en matière de séparation avaient donc amené les femmes à être reconnues titulaires de droits naturels, à savoir légitimement habilitées à revendiquer des prérogatives juridiques, parmi lesquelles il y avait la séparation d'avec le mari sans le consentement de l'Église, la demande de soutien financier aux exépoux, la possibilité de nommer leurs avocats respectifs. Bianca Premo conclut à ce propos que le recours important dans la seconde moitié du XVIII siècle des femmes aux tribunaux, comme celui des esclaves et des Indiens, contribua à produire une forme différente de Lumières. Au lieu de se limiter aux cafés et aux salons des élites intellectuelles, elles émergeaient du bas des sociétés coloniales. Grâce à la justice, ces groupes réussirent, selon elle, à imposer une nouvelle vision de la société et des relations sociales.

L'ample recours à la justice de personnes ordinaires à la fin de l'époque coloniale montre que les luttes pour la revendication des droits de citoyenneté ont eu lieu dans les empires avant de se transformer en révolutions contre ceux-ci ${ }^{31}$. Ceci nous permet de comprendre pourquoi les groupes subalternes, y compris les femmes, se sont mobilisés et ont participé aux mouvements des Indépendances.

\section{Le statut incertain des femmes à l'époque des Indépendances}

Le rôle acquis par les femmes à la fin de la période coloniale nous aide à comprendre l'incertitude quant à leur statut juridique et leurs droits pendant l'Indépendance. Bien que les constitutions de cette époque (celle de Cadix de 1812 et celles publiées dans les territoires américains pendant les guerres) faisaient référence à un citoyen essentiellement masculin, excluant implicitement les femmes de la citoyenneté, cette exclusion n'était pas si évidente ni absolue dans la pratique.

En premier lieu, dans les textes constitutionnels, il n'y avait pas une exclusion claire et nette des femmes des droits de citoyenneté.

\footnotetext{
$30 \quad$ Premo $2017: 151$.

31 Burbank \& Cooper $2010: 10$.
} 
Généralement, les conditions pour être reconnu comme citoyen étaient d'" être un homme libre, vecino, père ou chef de famille, avec une maison et des revenus sans aucune dépendance de quelqu'un d'autre ${ }^{32}$. Or, même si les termes sont tous déclinés au masculin, comme on l'a déjà souligné, vecino signifiait être membre d'une communauté et jouir d'une réputation sociale. Sur la base de cette conception locale et négociée de l'inclusion politique, le régime libéral espagnol et ceux qui s’imposent en Amérique pendant et après les indépendances ont élaboré un concept très ouvert et flexible de citoyenneté. Sa définition s'appuyait sur des critères locaux, moraux et subjectifs (" un style de vie honnête ») plutôt que sur des critères précis définis par le haut.

Pendant l'application de la constitution de Cadix en Amérique, il y eut divers recours des autorités locales aux Cortès demandant si les femmes pouvaient être admises au vote en tant que vecinas. En effet, il n'était pas du tout exceptionnel que les veuves ou les femmes séparées soient considérées chefs de famille et partant vecinas. Comme certaines femmes avaient voté par le passé à l'occasion des élections du conseil municipal, une autorité locale demanda aux tribunaux chargés d'appliquer la constitution, si elles pouvaient être admises au vote dans les nouvelles élections libérales. À Quito, les autorités supérieures répondirent que, sous la constitution de Cadix, les hommes votaient comme individus et non comme chefs de famille; par conséquent les femmes, même si elles étaient chefs de famille, n'avaient pas droit au vote ${ }^{33}$. En réalité, cette réponse contredisait le principe par lequel les femmes avaient été exclues des droits politiques, à savoir la condition de dépendance par rapport au pater familias. Cette ambiguïté autour des droits des femmes explique pourquoi il n'est pas difficile de retrouver quelques noms de femme dans les registres électoraux de cette période. Cela n'est pas surprenant si on considère le nombre de femmes qui étaient restées veuves pendant les guerres d'indépendance.

32 Article 2, titre 9 de la Constitución Politica del Estado de Cartagena de Indias (1812), disponible en ligne :

http://www.banrepcultural.org/sites/default/files/89670/brblaa169729.pdf (consulté le 23 février 2021)

33 Archives nationales de l'Équateur, fonds spécial, caisse 196, vol. 483, doc. 152. 
Une autre trace importante de la participation politique des femmes pendant l'indépendance est leur signature dans les registres qui ratifièrent la déclaration d'indépendance du Chili en 1818, comme le démontre le dernier livre de Lucrecia Enríquez. Même si la déclaration fut proclamée par O’Higgins, elle fut plus tard ratifiée par la majorité des villes et villages du Chili à travers des registres qui contenaient les signatures de vecinos. Dans le cas de Talca, au sud de Santiago, le registre incluait aussi les signatures de onze femmes, la plupart appartenant à la même famille ${ }^{34}$. Le registre fut envoyé à Santiago comme preuve de l'adhésion de la ville à la déclaration d'indépendance sans que personne ne songe à s'opposer aux signatures de femmes, évidemment considérées citoyennes au même titre que les hommes. Exprimant le consentement des pueblos à la déclaration, ces registres affirmaient implicitement leur participation active à la construction du nouvel État.

Sur la base de ces expériences, ce n'est pas un hasard si certains hommes politiques ont revendiqué les droits politiques pour les femmes. Au Mexique, en 1820, au milieu de l'explosion des pamphlets qui accompagnèrent la restauration de la constitution de 1812, après la rébellion de Rafael Riego en Espagne de 1820, certains écrivains, comme Joaquín Fernández Lizardi, affirmèrent clairement que les femmes non seulement auraient dû participer aux débats parlementaires, mais que, citoyennes, elles devaient être capables de voter et d'être éligibles et élues. De manière significative, il affirmait qu'on ne pouvait pas « leur attribuer un statut qui soit pire que celui de personnes originaires de l'Afrique, qui avaient été déclarées citoyens de l'empire comme quiconque $»^{35}$.

Ces discours publiés dans la presse ou dans les pamphlets, illustrent bien l'éventail des possibilités que le nouvel ordre politique, se fondant sur le principe d'égalité, avait ouvert non seulement pour les Indiens et les descendants d'Africains, mais aussi pour les femmes. Ce que Lizardi et d'autres intellectuels de l'époque voulaient souligner, c'était la contradiction entre le radicalisme de la révolution libérale et la continuité de certaines pratiques d'Ancien Régime,

\footnotetext{
34 Enríquez 2018 : 76.

35 Lizardi 1821, cité par Pani $2006: 11$.
} 
comme l'exclusion des femmes de la citoyenneté. En continuité avec le texte de Lizardi, un pamphlet intitulé l'Americana Constitucional affirmait que le régime constitutionnel de Cadix avait détruit toutes les inégalités. Étant donné que « la femme américaine était un patriote raffiné », elle était aussi « un citoyen et un vrai citoyen » et elle n'était pas «le sujet ou le serf de quelqu'un». En tant que bon citoyen, la femme américaine était née "pour obéir et gouverner, écrire et déclarer ses droits $»^{36}$.

\section{Droits et citoyenneté dans la République}

Contrairement à ceux qui affirment que l'Indépendance n'entraina pas de transformations significatives pour les femmes hispanoaméricaines, ces expériences et ces débats démontrent plutôt le contraire. Les recours aux tribunaux pour la reconnaissance de leurs droits, aussi bien que leur participation politique au cours de ces années, changèrent radicalement leur statut par rapport à l'époque coloniale. Cela est évident si on analyse les recours judiciaires de certaines femmes après l'indépendance, comme ceux étudiés par Arlène Díaz au Venezuela. Ici les femmes, comme les gens de couleur, adoptèrent les notions de liberté, égalité, citoyenneté garanties par la Constitution, les utilisant dans les tribunaux pour défendre leurs droits individuels.

Par exemple, en 1836, Micaela Ravelo y Alcántara fit appel au maire de Caracas pour obliger son mari à lui restituer la propriété qu'elle avait héritée de son père. Elle avait été mariée avec Juan José Espinosa pendant cinq ans, duquel elle avait eu cinq enfants. Juan José l'avait cependant abandonnée sans lui assurer de soutien financier; elle se plaignait donc de vivre dans la misère en dépit du fait qu'elle ait hérité d'un patrimoine. Son époux non seulement ne l'autorisait pas à utiliser l'argent hérité, mais il soutenait de plus qu'elle n'avait pas le droit de faire appel à un tribunal car le premier juge d'une épouse était son mari ; par conséquent elle aurait dû demander son autorisation au préalable. Il lui refusa donc son soutien.

36 Respuesta de la Americana Constitucional al Amigo de la Verdad, México, 1820, p. 3, cité par Pani $2006: 12$. 
Micaela répondit que Juan José n'était pas autorisé à administrer son patrimoine par la loi puisqu'elle ne lui avait pas cédé ses pouvoirs sur ses biens paraphernaux ${ }^{37}$; par conséquent :

[...] la constitution de l'État, qui me donne la précieuse condition de citoyen, me fait titulaire de mes droits et m'assiste dans la gestion de mes propriétés [...] Bien que je sois mariée, je ne suis pas pour ce motif l'esclave de M. Espinosa, je n'ai pas non plus renoncé à mes droits de citoyenneté, je reste propriétaire de mon patrimoine, je garde les libertés que les femmes de ma condition ont pour allouer ou non leurs propriétés à leurs maris, sauf les biens liés à la $\operatorname{dot}^{38}$.

Micaela fonde ses arguments sur les nouvelles lois républicaines qui garantissaient le droit de propriété aux citoyens : elle se considérait comme une citoyenne car elle était propriétaire.

Puisque Micaela appuyait son droit à gérer son patrimoine sur les principes constitutionnels, son époux dénonçait au contraire le caractère subversif de ces derniers. Mettant en question le contrôle familial, qui était la base de la société civile, affirmait Juan José Espinosa, ces principes auraient amené à l'anarchie. En outre, de tels principes défiaient son autorité et son honneur de mâle dans la société : un appel en justice fait par une femme contre un homme était, selon lui, un défi envers l'honneur masculin. L'époux de Micaela était donc très conscient des effets que les revendications des femmes, comme celles de son épouse, avaient pu créer dans la société.

Dans ce cas le discours traditionnel du mariage comme une forme d'esclavage s'enrichit des discours modernes sur la liberté et l'égalité. Ces femmes considéraient le mariage comme un partenariat contractuel, basé sur des obligations mutuelles. Pour elles, la position de père de famille était donnée aux hommes comme représentants de la famille; il ne s'agissait pas d'un privilège qui leur permettait de traiter les membres de la famille comme esclaves ou d'utiliser les patrimoines à leur bénéfice. Cela émerge clairement dans le recours de María Antonia Pérez, une autre femme qui attaque son mari pour des questions de propriété :

37 Biens possédés par une femme mariée sans faire partie de sa dot et qu'elle peut administrer à sa convenance.

38 Díaz $2004: 152$. 
Dans le mariage, les époux ne sont que les représentants de la famille et les femmes leurs partenaires qui devraient les aider avec les obligations maritales, mais qui ne sont pas condamnées à être les esclaves du mari ; les femmes ne sont pas non plus d'une condition inférieure dans l'exercice de leurs droits et la qualité de femme ne les exclut pas de l'ordre social ni de la garantie de jouir de la liberté et de la sûreté de leur personne. La loi nous rend tous citoyens égaux et si mon mari est autorisé à se comporter de manière libre, au même titre je suis autorisée à faire face moi-même à mes besoins ${ }^{39}$.

La défense de son patrimoine et de son bien-être entraîna une forte critique du comportement de son mari, affirmant en même temps son accès aux droits de citoyenneté réservés aux hommes. Puisqu'elle possédait les mêmes droits que les hommes, sa personne et sa propriété étaient protégées par la loi. Comme le révèle Christine Hünefeldt, les mêmes arguments furent utilisés par les femmes qui faisaient appel à la justice à Lima au début du XIX ${ }^{\mathrm{e}}$ siècle ${ }^{40}$.

Contrairement aux femmes de classe supérieure, comme Micaela, celles des groupes populaires démontraient leurs valeurs libérales en défendant leurs libertés individuelles à travers leurs comportements, en attendant que les lois protègent ces mêmes libertés. En raison de leur situation financière, ces femmes étaient obligées de travailler et de vivre de leur travail; cela leur permettait d'avoir une indépendance économique et sociale plus importante que les femmes des classes élevées. Certaines défendirent devant les tribunaux civils le droit de gagner un salaire. Par exemple, en 1836, Cecilia Barraniz, une actrice de théâtre de Caracas avait demandé le divorce pour cause d'abus commis par son mari. Dans son appel à la justice, elle déclara qu'elle avait toujours supporté son époux, mais qu'il n'avait jamais accompli ses devoirs. En outre, ce dernier voulait qu'elle abandonne sa profession. Cecilia demanda donc au tribunal d'obliger le mari à la maintenir ou bien de lui permettre de continuer son travail ${ }^{41}$. Pour ces femmes, il était essentiel que les partenaires ou maris respectent les obligations d'une union. Elles voulaient être traitées comme des citoyennes et être protégées par la loi comme les hommes.

\footnotetext{
39 Díaz 2004 : 168.

40 Hünefeldt $2000: 261,362$.

41 Díaz $2004: 167$.
} 
En 1835, une femme libre de couleur de Caracas, Barbara Jedler, dénonça au tribunal les abus commis par le maitre de son fils, M. Huizi, contre ce dernier. Ce faisant, elle demandait également que ses droits de femme libre soient respectés par le maittre :

Lorsque j'ai commencé à me battre contre un homme riche, puissant et influent, que pouvais-je m'attendre étant une humble femme de couleur et lui un homme d'une classe élevée et propriétaire de mon fils ? Quel pouvait être le résultat d'une controverse entre personnes de sphères différentes; et quel pouvait être mon succès si je ne pouvais pas compter sur la justice et l'impartialité du tribunal ? Cette persuasion m'encourage et l'égalité des droits assurée à tous les Vénézuéliens, me donne plus de force me permettant de ne pas cacher mon visage face aux arguments de M. Huizi. [...] Malgré ma condition d'esclave libérée, il méprise ma condition de femme respectable face aux autres hommes et à la société, mais il ne devrait pas être cru car je peux utiliser mes droits ${ }^{42}$.

Le recours contre les abus commis par le maittre envers son fils, encore esclave, permit donc à cette femme de couleur de revendiquer ses droits de personne libre qui jouissait des mêmes droits que les autres citoyens, y compris celui de faire appel au tribunal pour dénoncer un homme riche et puissant.

Comme les cas analysés le démontrent, il n'est pas si évident que les femmes aient été nettement exclues de la citoyenneté pendant l'époque des révolutions en Amérique espagnole. Les femmes ont un recours accru aux tribunaux dès la fin XVIII siècle et mobilisent le langage du droit naturel et de la citoyenneté avant de participer activement aux batailles des révolutions et des Indépendances. Avec les esclaves, les libres de couleur et les Indiens, elles participent à définir et à faire évoluer les catégories qui les concernent, ouvrant des espaces de participation politique impensables avant la rupture avec l'Espagne.

Pour les Hispano-Américains la définition horizontale de la citoyenneté avait la priorité sur sa définition verticale : pour eux, le statut de citoyen continuait à être défini par la société locale et non par l'État, à travers les recensements ou les registres des électeurs. Ce

42 Díaz 2004 : 169. 
trait fut ensuite normalement confirmé par les normes constitutionnelles qui, au XIX siècle, donnaient aux assemblées paroissiales le pouvoir de déterminer, sans possibilité d'appel, ceux qui étaient citoyens et ceux qui ne l'étaient pas. Par conséquent si une société locale pensait que certaines personnes d'origine africaine ou de genre féminin étaient des membres respectables de la communauté, elles étaient incluses dans la citoyenneté.

$\mathrm{Si}$ au lieu de regarder les grands mouvements féminins de protestation ou la participation des femmes dans les hautes sphères publiques, les historiens se focalisaient sur des expériences plus souterraines et moins évidentes de lutte sociale et politique, ils se rendraient compte qu'il y eut un changement considérable quant au régime de genre dans l'Amérique espagnole à l'époque des révolutions. Dans quelle mesure ce changement fut répandu et durable, voilà ce qu'il reste à explorer dans les sources judiciaires des archives locales et provinciales pour évaluer l'impact de cette transformation dans le temps et dans l'espace.

En même temps, l'idée selon laquelle la citoyenneté avait à cette époque une signification univoque et définie de façon rigide par l'État devrait être abandonnée. Au contraire, comme on l'a vu ici, l'action des femmes devant les tribunaux changeait continuellement son sens. La citoyenneté était une source des droits, une base pour des revendications, un symbole d'appartenance, plutôt qu'un statut clair et défini. C'était la possibilité de réclamer des droits ou mieux le droit d'avoir des droits en tant que membre d'une communauté, d'un État ou d'un empire.

\section{Bibliographie}

Arrom Silvia Marina, 1985, The Women of Mexico City, 1790-1857, Stanford, Stanford University Press.

BLANCHARD Peter, 2008, Under the Flags of Freedom: slave soldiers and the wars of independence in Spanish South America, Pittsburgh, University of Pittsburgh Press.

BurbanK Jane \& Frederick COOPER, 2010, Empires in World History: power and the politics of difference, Princeton-Oxford, Princeton University Press. 
Carranza Adolfo P., 1910, Patriotas argentinas, Buenos Aires, Sociedad Patriotas Argentinas.

Cartula Brú Victoria de, 1945, La Mujer en la independencia de América, La Habana, Biblioteca Historia, Filosofía y Sociología.

CHAMBers Sarah C., 1999, From Subjects to Citizens: honor, gender, and politics in Arequipa, Peru, 1780-1854, University Park, Pennsylvania State University Press.

—, 2006, "Masculine virtues and feminine passions: gender and race in the Republicanism of Simón Bolívar », Hispanic Research Journal, 7/1, p. 21-40.

Сотт Nancy F., 1998, "Marriage and women's citizenship in the United States, 1830-1934 », American Historical Review, 103/5, p. 1440-1474.

DE LA Fuente Alejandro \& Ariela Gross, 2010, «Comparative studies of law, slavery, and race in the Americas ", Annual Review of Law \& Social Science, 6, p. 469-485.

Diaz Arlene 2004, Female Citizens, Patriarchs, and the Law in Venezuela, 17861904, Lincoln, University of Nebraska Press.

Dore Elizabeth \& Maxine Molyneux (eds), 2000, Hidden Histories of Gender and the State in Latin America, Durham-London, Duke University Press.

DudinK Stefan \& Karen Hagemann, 2004, "Masculinity in politics and war in the age of democratic revolutions, 1750-1850 », in Stefan DUDINK, Karen Hagemman \& John TosH (eds), Masculinities in Politics and War: gendering modern history, Manchester, Manchester University Press, p. 3-21.

ENRIQUEZ Lucrecia, 2018, Historia, memoria y olvido del 12 de febrero de 1818. Los pueblos y su declaración de la independencia de Chile, Buenos Aires, Prohistoria.

FLETCHer Lea, 1994, Mujeres y cultura en la Argentina del siglo XIX, Buenos Aires, Editora Feminaria.

Franco Jean, 1989, Plotting Women: gender and representation in Mexico, New York, Columbia University Press.

GómEz Alejandro E., 2008, «La Revolución de Caracas desde abajo », Nuevo Mundo Mundos Nuevos, 8 [En ligne] [http://journals.openedition.org/nuevomundo/32982]

Helg Aline, 2004, Liberty and Equality in Caribbean Colombia 1770-1835, Chapel Hill-London, University of North Carolina Press.

Herzog Tamar, 2003, Defining Nations: immigrants and citizens in early modern Spain and Spanish America, New Haven, Yale University Press. 
HuFton Olwen, 1992, Women and the Limits of Citizenship in the French Revolution, Toronto, University of Toronto Press.

HÜNEFELDT Christine, 2000, Liberalism in the Bedroom: quarrelling spouses in nineteenth-century Lima, University Park, Pennsylvania State University Press.

JoHnSOn Lyman L. \& Sonya LiPSETT-Rivera (eds), 1998, Sex, Shame and Violence: the faces of honor in colonial Latin America, Albuquerque, University of New Mexico Press.

Kerber Linda K., 1998, No Constitutional Right to Be Ladies: women and obligations of citizenship, New York, Hill \& Wang.

KLUEGER Viviana, 2003, Escenas de la vida conyugal: los conflictos matrimoniales en la sociedad virreinal rioplatense, Buenos Aires, Editorial Quorum-UMSA.

LANDES Joan, 1992, Women and the Public Sphere in the Age of French Revolution, Ithaca, Cornell University Press.

LASSO Marixa, 2007, Myths of Harmony: race and republicanism during the age of revolution, Colombia 1795-1831, Pittsburgh, University of Pittsburgh Press.

LAVRIN Asunción (ed.), 1978, Latin American Women: historical perspectives, Westport-London, Greenwood Press.

-, 1984, "Women in Spanish American Colonial Society», in Leslie BeTHell (ed.), Cambridge History of Latin America, vol. II, Cambridge, Cambridge University Press, p. 321-355.

—, 1989, Sexuality and Marriage in Colonial Latin America, Lincoln \& London, University of Nebraska Press.

LiZARDi Joaquín Fernández de, 1821, Cincuenta preguntas del Pensador a quien quiera responderlas, México, Imprenta imperial de D. Alejandro Valdés.

Masiello Francine, 1992, Between Civilization and Barbarism: women, nation, and literary culture in modern Argentina, London-Lincoln, University of Nebraska Press.

Morelli Federica, 2020, Free People of Color in the Spanish Atlantic: race and citizenship, New York, Routledge.

ORTIZ ESCAMILLA Juan, 2014, Guerra y Gobierno. Los pueblos y la independencia de México, 1808-1825, México, El Colegio de México.

PANI Erika, 2006, " "Ciudadana y muy ciudadana"? Women and the State in Independent Mexico, 1810-1830», Gender \& History, 18/1, p. 5-19.

PREMO Bianca, 2017, The Enlightenment on Trial: ordinary litigants and colonialism in the Spanish Empire, New York, Oxford University Press. 
RABINOVICH Alejandro, 2013, Ser soldado en las Guerras de Independencia. La experiencia cotidiana de la tropa en el Río de la Plata, 1810-1824, Buenos Aires, Sudamericana.

RAMíreZ María Imelda, 2000, Las Mijeres y la sociedad colonial de Santa Fé de Bogotá 1750-1810, Bogotá, Instituto Colombiano de Antropología e Historia.

RODRÍGUEZ SÁENZ Eugenia, 2000, Hijas, novias y esposas: familia, matrimonio y violencia doméstica en el Valle Central de Costa Rica, 1750-1850, Heredia, Editorial Universidad Nacional.

RosanVALLON Pierre, 1992, Le Sacre du citoyen. Histoire intellectuelle du suffrage universel, Paris, Gallimard.

SÁBATO Hilda, 2018, Republics and the New World: the revolutionary political experiment in 19th-century Latin America, Princeton, Princeton University Press.

SCully Pamela \& Diana PATON (eds), 2005, Gender and Slave Emancipation in the Atlantic World, Durham-London, Duke University Press.

Seed Patricia, 1998, To Love, Honor, and Obey in Colonial Mexico, Palo Alto, Stanford University Press.

Socolow Susan M., 2000, The Women of Colonial Latin America, Cambridge, Cambridge University Press.

SORIANO Cristina, 2018, Tides of Revolution: information, insurgencies, and the crisis of colonial rule in Venezuela, Albuquerque, University of New Mexico Press.

URIBE-URÁN Víctor M., 2000, Honorable Lives: lanyers, family and politics in Colombia, 1780-1850, Pittsburgh, University of Pittsburgh Press.

—, 2015, Fatal Love: spousal killers, law, and punishment in the late colonial Spanish Atlantic, Palo Alto, Stanford University Press.

THIBAUD Clément, 2003, Repúblicas en armas: los ejércitos bolivarianos en la guerra de independencia en Colombia y Venezuela, Bogotá, Planeta [République en armes. Les armées de Bolivar dans les guerres d'indépendance du Venezuela et de la Colombie, Rennes, Presses Universitaires de Rennes, 2006]

ZAHLER Reuben, 2015, « ¿Y para las damas qué? Liberalism, nationalism and gender in the Hispanic world", in Scott EASTMAN \& Natalia SOBReVILLA PEREA (eds), The Rise of Constitutional Government in the Iberian Atlantic World, Tuscaloosa, The University of Alabama Press, p. 212-244.

ZegarRa Margarita (dir.), 1999, Mujeres y género en la historia del Perú, Lima, Centro de Documentación sobre la Mujer. 